\title{
IoT and Blockchain in the Development of Smart Cities
}

\author{
Laith T. Khrais \\ Department of Business Administration, College of Applied Studies and Community Services \\ Imam Abdulrahman Bin Faisal University, Dammam, Saudi Arabia
}

\begin{abstract}
With the advent and proliferation of the internet, the fourth industrial revolution is in full swing. As a result, different technologies have the potential to impact the course of human development. In other words, worldwide populations are moving towards growing urban centers and as a result, smart cities are emerging as the integration of human activities and technologies. These smart cities are built on top of different technologies such as blockchain and the Internet of Things (IoT). Consequently, the applications of these technologies in current and future smart cities will not only change the nature of human interaction and governance but also how business is conducted. This paper proposes an experimental study (qualitative and quantitative) that will determine the impact of blockchain and IoT technologies on the development of smart cities. It aims to derive insight from questions such as how current business models are preparing themselves for this disruption, the challenges they will face, and the potential contributions the two technologies will have on business development. The study's outcomes will provide the rationale for why businesses should start paying attention to these technologies and start on an early adoption plan that will slowly transform their business models as smart cities mature.
\end{abstract}

Keywords-Internet of things; blockchain technologies; smart cities; emerging markets; electronic commerce

\section{INTRODUCTION}

Powered by technologies such as the internet, the fourth industrial revolution is coming along exponentially as the world transforms into a global village. As a result, new markets are emerging, such as smart cities that use technologies such as online payment, internet connectivity, and blockchain, among others, to perform transactions in a fast and secure manner [1]. Besides, according to Schwab and Davis [2], the fourth industrial revolution is powered by data and, therefore, any technology that is data intensive such as the Internet of Things (IoT), cloud computing, machine learning and, artificial intelligence will not only grow in value but be applied widely. It should be noted that IoT in this paper will not only include embedded devices with network connectivity but also other portable computing platforms such as tablets, laptops, and smartphones.

As a result, connectivity is a very important aspect of smart cities where wireless connectivity via the Wi-Fi protocol is not only available from the devices people use in their daily lives but also provided by internet and network service providers [3]. Additionally, people use this connectivity to connect as well as shop online and do their banking at the convenience of their homes or workplaces. Other online to offline interactions also occur where, for example, an individual can purchase a CCTV surveillance camera for their home or business and get after-sale services such as installation and maintenance for free depending on the vendor and location.

These smart cities, however, are built around centralized infrastructures and, as a result, a vulnerable to a single point of failure attack. A bank, for example, can charge very high tariffs for every transaction at the cost of the convenience. Additionally, the bank may not always be online or might be breached thus losing customer data to malicious attackers, as evidenced by the recent Capital One data breach [4]. Additionally, with the growing demand for personal data such as credit card information, passport details, and medical history on the dark web [5], smart cities expose their residents to undue risks.

To solve these and other problems facing smart cities, blockchain technology, derived from the bitcoin paper by Satoshi Nakamoto [6], is needed. Not only are blockchains tolerant to attacks, but they are also tolerant of failure (Byzantine fault tolerance), decentralized, secure, and peer to peer atomic transactions that eliminate the man in the middle (and their tariffs) [7]. When the decentralized nature of blockchains is coupled with the versatility of IoT, smart cities get the potential to grow further while shoring up their weaknesses.

There are drawbacks to using blockchain technologies in smart cities, however. As decentralized systems, blockchains exist outside the regulation and control of governments and their regulatory bodies. As a result, some countries have banned blockchains such as bitcoin while they view other decentralized applications (DApp) as threats [8]. Therefore, this paper is a review and exposition of blockchain and IoT technologies in the development of smart cities. As a result, the paper is divided into three major sections. The first section will review all background information, such as the emerging markets in smart cities, their business models, and their impact. The second section will conduct a qualitative and quantitative study on the development of blockchain and IoT technologies smart cities while the last section collects the results and discusses the findings.

\section{LiterATURE REVIEW}

\section{A. Survey of Emerging Markets and Smart Cities}

The 90s ushered in a period of unprecedented development with the invention and proliferation of electrical and electronic household and communications devices such as computers, 
modems, cable tv, and the earliest predecessor of the modern internet powered by the world wide web (and HTTP) [3]. This trend has developed and created new markets such as ecommerce, social networking, online shopping, and advertisements. These technologies have enabled companies to derive over $60 \%$ of their revenue from online activities such as advertisements [9] [10]. In 2014, for example, e-commerce sales ballooned from 6 billion dollars of previous years to over 25 trillion dollars and counting [1]. Therefore, the potential and opportunities for the growth and development of smart cities already exist. All that is missing are the underlying technologies and their associated business models to get the ball rolling.

\section{B. Preview of the Two Business Models}

This paper looks into the application of two business models, namely the Internet of Things (IoT) and Blockchain, and their interaction with the models of operations of ecommerce in smart cities. Besides, while blockchain and IoT can be implemented independently, their integration gives a product that is more than the sum of the individual technologies. This section outlines the existing business model built on top of IoT and Blockchain while citing successful examples.

1) Internet of Things (IoT) business models: In a nutshell, IoT is the systematic method of attaching sensors to an existing system and getting data out of the system. As of 2015, the IoT industry was valued at 11 trillion dollars, but despite this, three out of four IoT businesses fail [11]. Despite this, IoT devices proliferate cities and homes from smart alarms to refrigerators and TVs [12]. The reason behind this high failure rate is that most individuals and companies who apply IoT in their businesses get access to the data but do not know how to turn it into a source of competitive advantage or revenue [12]. Similar to how data is the currency for the fourth industrial revolution [2], data is the backbone of IoT and its associated business models. The following paragraphs present the different business models built on top of IoT.

Industries such as aerospace, oil, and gas extraction are heavily regulated due to the high risks, costs of accidents occurring, and the widespread consequences the accidents would have on the social, political, environmental, and economic aspects of commercial operation. The traditional method of ensuring safety was to set compliance standards, and having human inspectors regularly inspect the equipment and plants [13]. With IoT, however, businesses ensure compliance by attaching sensors where plant monitoring can be done in real-time [14]. As a result, companies can be proactive about safety without incurring additional costs. This practice has become common in the aerospace industry, where jet engines are equipped sensors that transmit engine health data to the manufacturer for real-time monitoring, performance evaluation, and warning [15].

The second business model applying IoT is preventative maintenance, where cheap IoT platforms can be used to monitor systems and plants in operation continuously and warn when malfunctions occur due to unfavorable operating environments [16]. Alternatively, equipment or plants can be scheduled for regular maintenance schedules where they are shut down. However, the economic costs of these downtimes can be prevented by using preventative maintenance policies, especially in equipment and plants whose operation and maintenance are complex [16]. General Electric has successfully utilized preventative maintenance with IoT enabled sensors in the wind turbine blades to not only change the pitch angle depending on the local airflow but also schedule downtimes for maintaining the turbines when there is no wind blowing [12].

Last but not least, IoT sensors and systems have found widespread application in the field of remote diagnostics, especially in the medical and agriculture industries. Greenhouse operators, for example, use IoT sensors to remotely monitor and control favorable plant growing conditions such as humidity, light, temperature, and $\mathrm{CO}_{2}$, among others [17]. In the medical industry, IoT platforms have found application in patient condition monitoring (using wearable devices), treatment (such as insulin pumps), and fitness tracking [18].

These IoT business models assume that the users and businesses rely on their expertise to design, develop, and produce these IoT platforms (both hardware and software). The truth, however, is that different business models have emerged that are catered to providing technical know-how where they develop the products the end users build their businesses on [19]. An IoT device developer and provider might, for example, lease out their products (especially software that runs on dedicated cloud servers) on a subscription model and make support services available 24/7.

Similar to the Software as a Service (SaaS) cloud architecture and business model, the subscription model has the advantage of sustainable revenue instead of selling the product in a one-time sale then having the customers pay for future upgrades [20]. Alternatively, an IoT developer and manufacturer might opt not to sell their product but sell the desired outcome. This outcome-based business model focuses on meeting the customer's needs instead of making a product that a customer might choose to fit their needs. Rolls Royce is such an example where they sell the service (24/7 engine health and performance monitoring for every engine they sell, and it is in service) instead of simply selling engines based on the aircraft manufacturer's or operator's requirements [15].

2) Blockchain business model: The downside of smart cities is that there can be too much connectivity that instead of being a boon or extra feature, it starts to become an anchor. Take the internet; for example, targeted advertisement has become so common that companies create profiles of their users and sell the data to other companies. Additionally, user and browser fingerprinting have become advanced that it is now entering ethical grey areas [21]. In IoT, different devices and sensor networks collect private, confidential, and business secrets that are vulnerable to exposure through attacks such as a man in the middle attack [22]. Besides, even trust in the devices themselves is not guaranteed, as evidenced by the actions of the mobile phone company Huawei and the Chinese government [23]. 
As a result, the integration of blockchain and IoT will shore up the deficiencies of smart cities. However, what is blockchain? A blockchain is a distributed ledger whose entries are time-stamped and cryptographically signed to ensure their immutability [24]. A business model built on top of blockchain technology will not only become decentralized (exhibiting Byzantine fault tolerance) but also peer to peer transaction in a network where trust is enforced by the business model [25].

As a result, decentralization, immutability, and transparency form the cornerstones of all blockchain-based business models [26]. These business models utilize the blockchain infrastructure in three primary ways. First, they store all their data on the blockchain to ensure that the data is tamper-proof [27]. Secondly, they apply the transparency feature of blockchains to enhance the functionality and utility of existing infrastructures such as supply chains [28]. Lastly, the more advanced business models build their artificial intelligence systems on top of blockchains to build a decentralized AI system [29]. The following paragraphs describe the different business models inspired by or built on top of blockchains.

The easiest business model for a non-technical company that wants to integrate blockchain technology into their existing infrastructure and business model is the Blockchain as a Service business model. This is because the ecosystem allows the business to create their products, experiment, and release their product while abstracting away low-level infrastructure details [30]. One example is the Ethereum Blockchain as a Service developed and managed by a joint venture between Microsoft and ConsenSys [31].

A utility token business model, on the other hand, is based on the use and exchange of tokens that have inherent value within the blockchain. This business model, also known as token economics, performs a similar function to traditional banks only that they are decentralized, and the creation, utilization, and destruction of tokens are not under the control of a central authority such as government [32]. Besides, the tokens themselves are valuable because the users deem them valuable and not because they are backed by gold reserves, for example. As a result, utility token business models become profitable only when the value of the individual tokens increase.

In contrast, a securities business model is a recent blockchain business model where a company sells tokens known as securities that are expected to gain value when individuals buy them. In other words, a blockchain security token is synonymous with possessing legal ownership to an asset [33]. The role of the blockchain is, therefore, to verify the real owners of the tokens as well as regulate the creation and consumption of security tokens. This business model also allows verified owners not only to trade their tokens but also use them as collateral. As a consequence, the securities business model has the potential to not only redefine the traditional concept of ownership but also provide a means of redistributing wealth among a large population without devaluing the tokens [34].

\section{Impact of the Business Models}

It is projected that the IoT industry will grow to encompass over 20 billion devices by 2020, 4.5 billion of them being from Europe [35]. Additionally, over 65\% of the worldwide human population is projected to have moved to urban cities by 2040 [35]. As a result, the emerging smart cities will not only be relying on traditional wireless networks to provide connectivity but also local area networks powered by the streetlight to form a mesh network or a wide area network (WAN). Besides, the exponential growth in the number of connected IoT devices in a smart city increases the risk of unauthorized access to private and sensitive data transmitted over the wide-area networks. It, therefore, becomes imperative that blockchain technology is used to secure these communications while maintaining transparency and data integrity. As a result, the following are how the integration of blockchain and IoT technologies would impact the development of smart cities.

IoT security is a concern that all developers and some end users recognize. This is because there have been demonstrations of how the security of IoT devices, especially the data they collect and transfer can be easily compromised. Such systems use traditional cryptographic methods of securing the data where private keys are exchanged and data transmitted over the network in the form of ciphertext. However, considering the global cost of cybercrime [36], the potential damage to smart cities would be exponential and can even destabilize a city. As a consequence, a different security architecture is needed where instead of an attacker breaking the security on singular devices, they will be forced to break the entire network (at least 51\% in case of a blockchain-based IoT network). Blockchain networks are very hard for a malicious attacker to gain control because they have an enormous price tag attached to such efforts. As a result, by securing IoT networks with blockchain technology, the developers and users can be safe from most attacks, except individuals or institutions that are willing to pay the price [37].

There is a catch, however. In its current state, blockchain technology is slow and not scalable [38]. For instance, on the bitcoin network, transactions are stored and verified in blocks before they are permanently added to the blockchain. On average, verifying transactions in a single block takes 10 minutes. For a city with billions of devices, the speed of the IoT network built on top of the blockchain infrastructure will drop. This will also impact business models using blockchain. As a result, businesses might be expected to do a cost-benefit analysis and decide to do away with the extra security that blockchain affords. Security-conscious users and developers, however, might find the speed as a worthwhile trade-off. Efforts are underway, however, to improve the speed of blockchain and make it scalable [39] [40].

Despite the drawbacks, blockchain and IoT will be the cornerstone of current and future smart cities. This is because the integration of the two platforms is highly versatile, as well as easy to implement. One such impact these two technologies will have in smart cities is how they will change public transit. Public transit ridership has been on the rise in the last two decades, where millions of workers and city residents use it to move from point A to B (such as home to work) [40]. The number is expected to rise given the growing trend of climate 
awareness and the efforts to minimize further pollution by reducing emissions from burning fossil fuel. The Maltese government, for example, has contracted the private company Omnitude to develop and provide solutions to the challenges facing its public transport sector using blockchain technology [41]. This system is expected to be a payment point for the different public transport systems to improve transparency in the operations and sector.

Besides, another element of smart cities to be impacted by IoT and blockchain is e-commerce. Built on these two technologies, smart cities of the future will enhance the provision of local products and services to residents close to their homes or workplaces. Such drive for encouraging local uniqueness will encourage residents to create products and services using blockchain and IoT, thus eschewing national and overly capitalistic brands in favor of local brands that serve the local population. For this to be successful, however, the different blockchain applications and IoT platforms will have to be interoperable and compatible with each other [42]. This challenge is currently being solved by companies such as Chain of Things that are attempting to make IoT (software and hardware) and blockchain infrastructures interoperable [43].

\section{Methodology}

This section presents the methodology and research design aimed to determine the impact of IoT and blockchain technologies in the development of smart cities. As a result, the research design will both qualitative and quantitative. While the quantitative aspect of the research will give insight into the statistical aspects of the study, the quantitative study will provide additional information, especially objective and subjective perspectives the research participants have on the subject matter.

\section{A. Qualitative Approach}

The qualitative approach will entail collecting secondary information on model companies and assessing it to address the research problem. Businesses that adopt the Internet of Things and blockchain models will constitute the population for this study. A majority of the companies to be sampled for the study is in the world's smart cities and upcoming markets. A random sampling technique will be used to prevent sampling and information bias in the findings of the study. The sampling method would take into consideration the different outplays that cannot be left out, including a review of the previous studies about and related to the subject matter.

The qualitative approach has the limitation that technologies are constantly evolving. As a result, the findings will not necessarily represent the current state of affairs in the industry. For this reason, secondary data is not adequately updated or, at times, is left unattended as brand new models eclipse the previous ones. For instance, smart cities and emerging markets show that the shoppers and bankers feature a high use of technology in the form of two business models (IoT, blockchain or a combination of both). The two models define ways of making the length of processing transactions shorter and more flexible and transparent. Charts and graphical representation will be applied to understand the concepts and derive the impacts on e-commerce while using such business models.

\section{B. Quantitative Approach}

Besides, the study will employ a quantitative approach to address the research problem. The approach will be useful in collecting information on the perspective of industry experts on the various developments and dynamics of blockchain technologies and the Internet of Things.

1) Target population: The study will target company managers and intellectuals from the industry, such as university professors, pioneers of IoT, and blockchain technologies. The participants will be drawn from various companies as well as from different sectors. Consequently, the study will recruit 60 participants to contribute to the required information. Although a sample size of 60 is not enough to substantiate the validity of the findings of the study, it is appropriate for a pilot study on a fact-finding mission.

2) Research instrumentation: The study will utilize interviews to collect quantitative data. The interviews will constitute both closed and open-ended questions. The closedended questions will gather statistical data that will enable the researcher to measure opinion and the popularity of ideas on the subject matter. On the other hand, the open-ended questions will help obtain detailed information on the questions through the explanations given by the participants.

3) Data analysis: The collected data will be recorded in tables and analyzed using Excel software. Using the Excel program, the researcher will derive graphical illustrations and charts from demonstrating the outcome of the study.

\section{Ethical Considerations}

This study will adhere to the conventional ethical considerations that govern research work. These include obtaining informed consent from the sampled and accepted participants of the study before starting the study while respecting their decision not to take part in the study for various reasons. Secondly, the participants of the interviews were briefed on the purpose of the study, and hence, were given a chance to exercise free-will to give the required information. Considering this, no participant was coerced to engage in the study. Finally, the participants were accorded due to confidentiality and anonymity, as their details such as names were not recorded anywhere in the course of the study where codes (alphanumeric) were used.

\section{RESUlTS AND DiscusSION}

After data analysis, the findings of the research will be discussed in this section. The following are the different topics (aspects) of the subject matter that will be investigated from the qualitative and quantitative research designs.

\section{A. Contributions of IoT and Blockchain Technologies to Business Development}

Fig. 1 shows the ratings for how different users would use the combination of IoT and blockchain, and what they preferred or would like improved about the integration of the two technologies as they are used to develop smart cities. 
These include faster, more scalable transactions, and transparency in the whole infrastructure (five-star ratings). On the other hand, IoT and blockchain technologies incorporate radio frequency identification to check on product quality, conditions and individual requirements. For instance, shoppers with an interest in chilled red meats or frozen dairy products can use such models to indicate their interests when placing orders. IoT, in combination with blockchain, helps product assessment to be conducted continuously.

Additionally, the end-to-end conversation between sellers and shoppers assists in real-time rectification of mistakes in product specifications, and the shoppers are, therefore, assured of high-quality products through the use of IoT and blockchain platforms. Besides, they are supplied with periodic updates on the service improvements and changes in the websites or devices to keep me up with the industry trends. Once again, at the backdrop of cutting short intermediaries, there can be the creation of jobs in the courier and warehouse services, in case deliverables are collected expressly.

These are among the multiple impacts of IoT and blockchain on the development of smart cities that will be substantiated by the data collected in this study. In this context, Fig. 2 and Fig. 3 summarize the fields impacted by blockchain and IoT as well as how their application is projected to integrate with communities living in smart cities.

However, there is a concern that such a research design is flawed and would be susceptible to confirmation bias. The conductors of this study have, however, considered this and prevented such an event by using statistical methods such as working by positing a null hypothesis to be confirmed or rejected by the collected data.

\section{B. The Readiness of Modern Businesses in Embracing IoT and Blockchain Technologies}

The interview questions will ask the participants whether their businesses have the capacity to adopt the Internet of Things and blockchain technology. Additionally, they will be asked if they are equipped with the resources necessary to not only be competitive enough in the emerging smart cities but also be profitable. This will help to understand questions on the readiness of the current business models to evolved and adapt to the changing tides. Out of 60 respondents, 25 noted that their companies were ready to fully pursue the transition. However, 35 of the respondents stated that the full embracement of the new changes requires undergoing structural and human resources adjustments in their organizations.

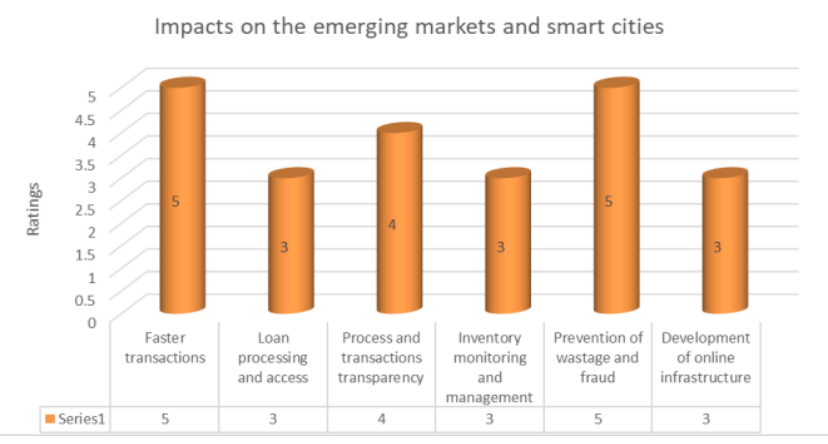

Fig. 1. Business Models and their Implications in Emerging Markets and Smart Cities.

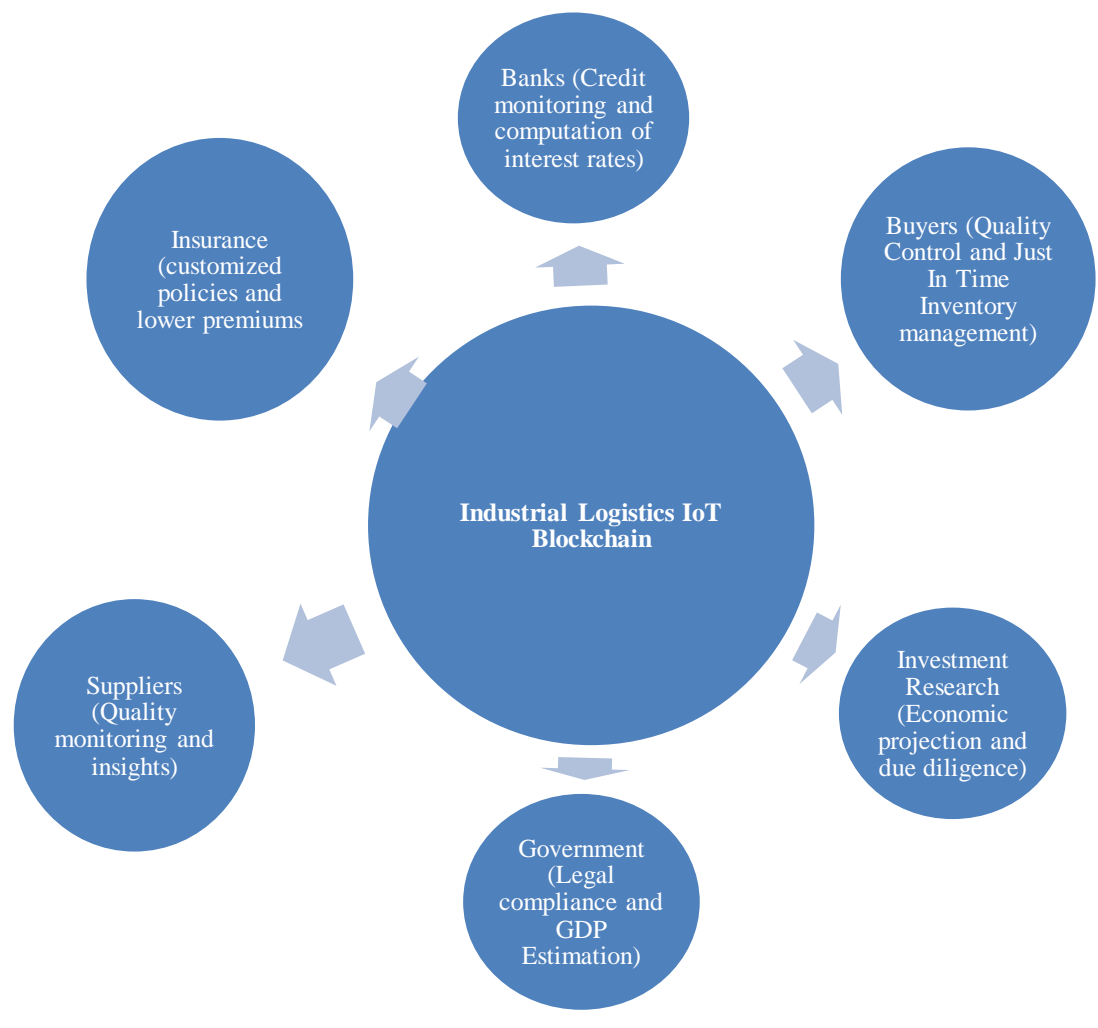

Fig. 2. The Analysis of the IoT Impacts on Business Commerce. 


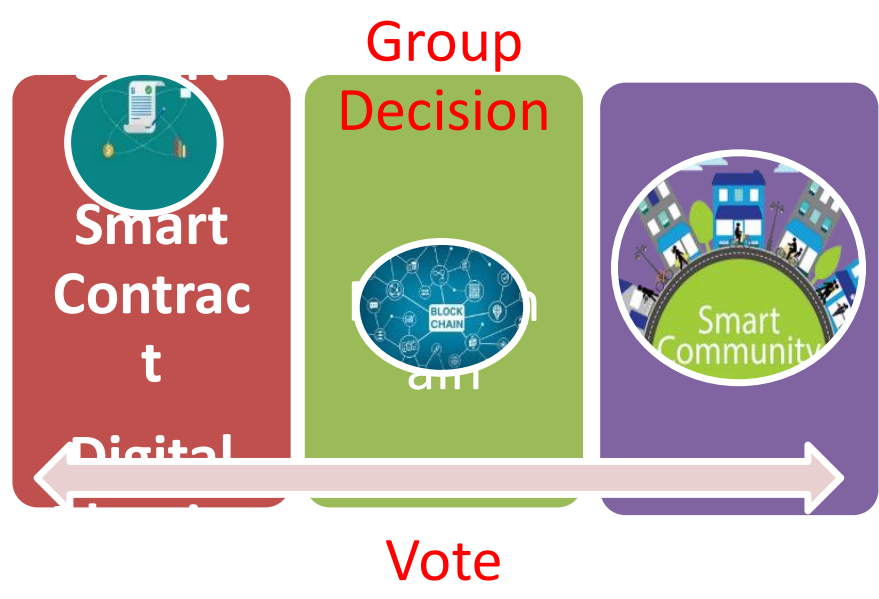

Fig. 3. Application of Blockchain in Emerging Markets.

\section{Challenges Encountered when Adopting IoT and Blockchain Technology}

The participants will also be prompted to indicate the challenges their companies encountered or were likely to encounter during enhancing of the IoT and blockchain technologies. This will help to determine which resources, such as personnel and different mindsets, are required in the transition and operation of the business models that integrate blockchain and IoT technologies. Additionally, the study aims to determine these challenges because they are also business opportunities for current and future entrepreneurs to exploit. These are summarized as follows in Fig. 4.

\section{Theoretical and Practical Contributions}

The outcome of this study will be imperative in giving information on the benefits of IoT and blockchain technology in businesses. Hence, this study establishes the rationale for companies to adopt the technologies. Besides, the study points out the challenges encountered by organizations in adopting the technology, thus, forming a foundation for further studies on the elimination of these hindrances to facilitate business development.

\section{CHALLENGES FACING THE ADOPTION OF IOT AND BLOCKCHAIN TECHNOLOGY}

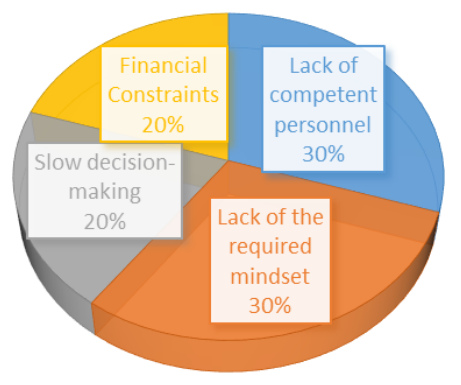

Fig. 4. Challenges Facing IoT and Blockchain.

\section{CONClusion}

The next step of development is smart cities, evidenced by the worldwide rural to urban migration, blockchain and IoT promise to deliver unprecedented development in all aspects of human society. Besides, the complementary nature of blockchain and IoT promises to solve most problems businesses face, especially in their adoption of new and untested business models. This paper proposed a study aimed to determine the developmental impact blockchain and IoT technologies will have on smart cities. Once complete, the research will reveal insight into issues such as the contributions of these two technologies as well as the challenges individuals and businesses will face when transitioning business models among others. Future studies will then build on this paper while expanding on the subject matter as well as larger sample sizes. Besides, time and financial constraints were very important factors in determining the study's sample size, despite its limitations.

\section{REFERENCES}

[1] L. G. Anthopoulos and P. Fitsili, "Understanding smart city business models: a comparison," in Proc. of the 24th International Conference on WorldWide Web. Florence, 2015. doi: 10.1145/2740908.2743908.

[2] Schwab, Klaus, and Nicholas Davis. Shaping the future of the fourth industrial revolution. Currency, 2018.

[3] J. Ruan and Y. Shi. Monitoring and assessing fruit freshness in IOTbased e-commerce delivery using scenario analysis and interval number approaches, Information Sciences, 2016,373(1), 557-570.

[4] Lu, Jack. "Assessing The Cost, Legal Fallout Of Capital One Data Breach." Legal Fallout Of Capital One Data Breach (August 15, 2019) (2019).

[5] Stack, Brian. "Here's how much your personal information is selling for on the dark web." (2018).

[6] Stifter, Nicholas, et al. "Agreement with Satoshi-on the formalization of Nakamoto consensus." (2018).

[7] O. Sohaib, L. Haiyan and W. Hussain. "Internet of Things (IoT) in ecommerce: For people with disabilities," in 2017 12th IEEE Conference on Industrial Electronics and Applications (ICIEA). Siem Reap, Cambodia, 2017. doi: 10.1109/ICIEA.2017.8282881.

[8] Kewell, Beth, and Peter Michael Ward. "Blockchain futures: With or without Bitcoin?." Strategic Change 26.5 (2017): 491-498.

[9] Liu, Stephanie Q., and Anna S. Mattila. "Airbnb: Online targeted advertising, sense of power, and consumer decisions." International Journal of Hospitality Management 60 (2017): 33-41.

[10] Aslam, Bilal, and Heikki Karjaluoto. "Digital advertising around paid spaces, E-advertising industry's revenue engine: A review and research agenda." Telematics and Informatics 34.8 (2017): 1650-1662.

[11] Manyika, James, et al. "Unlocking the Potential of the Internet of Things." McKinsey Global Institute (2015).

[12] Blanding, Michael. "The Internet Of Things Needs A Business Model. Here It Is". HBS Working Knowledge, 2019, https://hbswk.hbs.edu/item/the-internet-of-things-needs-a-businessmodel-here-it-is.

[13] Hopkins, Andrew. "Beyond compliance monitoring: new strategies for safety regulators." Law \& Policy 29.2 (2007): 210-225.

[14] Reza Akhondi, Mohammad, et al. "Applications of wireless sensor networks in the oil, gas and resources industries." 2010 24th IEEE International Conference on Advanced Information Networking and Applications. IEEE, 2010. 
[15] Royce, Rolls. The jet engine. John Wiley \& Sons, 2015.

[16] Chaudhuri, Arindam. "Predictive maintenance for industrial iot of vehicle fleets using hierarchical modified fuzzy support vector machine." arXiv preprint arXiv:1806.09612 (2018).

[17] Yu, Jinying, and Wei Zhang. "Study on agricultural condition monitoring and diagnosing of integrated platform based on the internet of things." International Conference on Computer and Computing Technologies in Agriculture. Springer, Berlin, Heidelberg, 2012.

[18] Almotiri, Sultan H., Murtaza A. Khan, and Mohammed A. Alghamdi. "Mobile health (m-health) system in the context of IoT." 2016 IEEE 4th international conference on future internet of things and cloud workshops (FiCloudW). IEEE, 2016.

[19] Fleisch, Elgar, Markus Weinberger, and Felix Wortmann. "Business models and the internet of things." Interoperability and Open-Source Solutions for the Internet of Things. Springer, Cham, 2015. 6-10.

[20] Tzuo, Tien, and Gabe Weisert. Subscribed: Why the Subscription Model Will be Your Company's Future-and what to Do about it. Penguin, 2018.

[21] Lerner, Adam, et al. "Internet jones and the raiders of the lost trackers An archaeological study of web tracking from 1996 to 2016." 25th \{USENIX\} Security Symposium (\{USENIX\} Security 16). 2016.

[22] Mahmoud, Rwan, et al. "Internet of things (IoT) security: Current status, challenges and prospective measures." 2015 10th International Conference for Internet Technology and Secured Transactions (ICITST). IEEE, 2015.

[23] Kwan, Martin. "Can Huawei Sue the US Government for Defamation? A Study on the Threshold of Foreign State Immunity from a Comparative Perspective." A Study on the Threshold of Foreign State Immunity from a Comparative Perspective (May 20, 2019) (2019).

[24] Puthal, Deepak, et al. "The blockchain as a decentralized security framework [future directions]." IEEE Consumer Electronics Magazine 7.2 (2018): 18-21.

[25] Hawlitschek, Florian, Benedikt Notheisen, and Timm Teubner. "The limits of trust-free systems: A literature review on blockchain technology and trust in the sharing economy." Electronic commerce research and applications 29 (2018): 50-63.

[26] Nowiński, Witold, and Miklós Kozma. "How can blockchain technology disrupt the existing business models?." Entrepreneurial Business and Economics Review 5.3 (2017): 173-188.

[27] Hwang, Junyeon, et al. "Energy prosumer business model using blockchain system to ensure transparency and safety." Energy Procedia 141 (2017): 194-198.

[28] Emmadi, Nitesh, and Harika Narumanchi. "Reinforcing Immutability of Permissioned Blockchains with Keyless Signatures' Infrastructure." Proceedings of the 18th International Conference on Distributed Computing and Networking. 2017.
[29] Salah, Khaled, et al. "Blockchain for AI: Review and open research challenges." IEEE Access 7 (2019): 10127-10149.

[30] Singh, Jatinder, and Johan David Michels. "Blockchain as a Service (BaaS): Providers and Trust." 2018 IEEE European Symposium on Security and Privacy Workshops (EuroS\&PW). IEEE, 2018.

[31] Onik, Md Mehedi Hassan, and Mahdi H. Miraz. "Performance Analytical Comparison of Blockchain-as-a-Service (BaaS) Platforms." International Conference for Emerging Technologies in Computing. Springer, Cham, 2019.

[32] Tasca, Paolo. "Token-Based Business Models." Disrupting Finance. Palgrave Pivot, Cham, 2019. 135-148.

[33] Tapscott, Don, and Alex Tapscott. Blockchain revolution: how the technology behind bitcoin is changing money, business, and the world. Penguin, 2016.

[34] Chen, Yan. "Blockchain tokens and the potential democratization of entrepreneurship and innovation." Business Horizons 61.4 (2018): 567575.

[35] Sallaba, M., D. Siegel, and S. Becker. "IoT powered by BlockchainHow Blockchains facilitate the application of digital twins in IoT." Deloitte Issue (2018).

[36] Morgan, Steve. "Cybersecurity Ventures predicts cybercrime damages will cost the world \$6 trillion annually by 2021." Cybersecurity Ventures (2017)

[37] Ali, Muhammad Salek, Koustabh Dolui, and Fabio Antonelli. "IoT data privacy via blockchains and IPFS." Proceedings of the Seventh International Conference on the Internet of Things. 2017.

[38] Chauhan, Anamika, et al. "Blockchain and scalability." 2018 IEEE International Conference on Software Quality, Reliability and Security Companion (QRS-C). IEEE, 2018.

[39] Otte, Pim, Martijn de Vos, and Johan Pouwelse. "TrustChain: A Sybilresistant scalable blockchain." Future Generation Computer Systems (2017)

[40] Eyal, Ittay, et al. "Bitcoin-ng: A scalable blockchain protocol." 13th \{USENIX\} symposium on networked systems design and implementation (\{NSDI\} 16). 2016.

[41] Magazine, Bitcoin. "Malta Government To Enhance Public Transportation Using Blockchain Technology". Nasdaq.Com, 2018, https://www.nasdaq.com/articles/malta-government-enhance-publictransportation-using-blockchain-technology-2018-05-17.

[42] Fraile, Francisco, et al. "Trustworthy industrial IoT gateways for interoperability platforms and ecosystems." IEEE Internet of Things Journal 5.6 (2018): 4506-4514.

[43] "Chain of Things". Chain of Things, 2020, https://www.chainofthings .com/. 